\title{
Petri Nets with Resources for modeling Primary Healthcare Systems
}

\author{
Cristian Mahulea $^{\dagger}$, Liliana Mahulea*, Juan-Manuel García-Soriano*, José-Manuel Colom ${ }^{\dagger}$ \\ *Servicio Aragonés de la Salud, Zaragoza, Spain \\ Email: \{lmahulea@gmail.com,jmgsoriano@movistar.es\} \\ ${ }^{\dagger}$ Dept. of Computer Science and Systems Engineering \\ University of Zaragoza, Spain \\ Email: \{cmahulea,jm\}@unizar.es
}

\begin{abstract}
Petri net is a powerful formalism for modeling, analysis and design of discrete event systems. With a good capacity of representing synchronization and concurrency they are used in different field of applications. This paper presents a modeling methodology of the primary healthcare system based on Petri nets. A disease of a patient is diagnosed and cured by following a sequence of treatments and cares belonging to a medical protocol. We show that for almost all of the diseases, each medical protocol can be modeled by a state machine Petri net. When the protocol is applied, different resources may be needed. Here we consider only resources that are conservative and they can be modeled by places where the number of tokens in the place says the number of available instances of that resource. By applying the available results of the Petri nets, many properties of the healthcare system can be studied. In particular, the prediction of different indices can be estimated by using the formal model.
\end{abstract}

Keywords-healthcare systems, Petri nets, modeling methodology

\section{INTRODUCTION}

An important indicator for the live quality in a country is the functionality and the results obtained by the health system. With a budged between 5 and $10 \%$ of the gross domestic product (GDP) it is one of the most important decision to make when the budget planning is done. Therefore, it is crucial to have a good prediction of its evolution. This prediction is not possible without a good formal model of the system.

In this paper we propose a modeling methodology for the healthcare system. Other methodologies have been proposed in literature. For example, in [1], [2] a stochastic mathematical programing model for operating room planning for surgery is presented. Using the Monte Carlo simulation and Mixed Integer Programming problems the solution is obtained. Petri net modeling has been considered in [3] where a continuous Petri net model of an emergency department is proposed. However, all these works consider only a particular problem of the healthcare system.

In [4] we proposed a modular Petri net modeling methodology for the health system. A health care area that is the basic entity of the health system can be seen as composed by different sub-systems called modules. This separation is done considering the different components of a health area. Each module can be modeled separately by a Petri net having some inputs and outputs modeled by places. The global model is obtained by fusion of these input and output places and by the addition of information on the population/patients. By applying this methodology, Petri Nets of the class $S^{4} P R$ are obtained, for which many properties are characterized through conditions based on the PN structure and the initial marking.

Differing by [4], in this paper we consider a modeling methodology by assuming the medical protocols that are used to cure a disease. Therefore, here we are not interested in the physical localization of the treatment or of the cure but only on the medical protocol. Moreover, only medical protocols used in primary healthcare system will be considered. To adopt a medical protocol, there are many resources that should be used in order to continue with the treatment. In this paper we consider only resources that are used in a conservative way (hence a model for a transplant surgery cannot be considered because the organ is not a conservative resource). However, we think that many medical protocols use only conservative resources.

The approach we propose for constructing the PN model of the health system assume a methodology in three steps:

(1) Patient flow and medical protocols. Each patient that enters in the system is an active process. Processes are modeled by tokens that pass through a $\mathrm{PN}, \mathcal{N}$, representing the medical protocol used to cure a particular disease. The places (local states) of $\mathcal{N}$ are related with different treatments or cures taken by the patients contained in these places. The transitions of $\mathcal{N}$ allow the progress of patients to their final states where it is assumed that they are cured or the disease controlled. A healthcare system has different input points of patients and output points of healthy patients corresponding to different medical protocols implemented. The execution of a medical protocol is achieved by the execution of a protocol path and several paths can exists in the same medical protocol corresponding to different decisions (according to the results of some medical tests/analysis) that should be taken during the implementation of the protocol. A protocol path is a sequence of transitions fireable in $\mathcal{N}$, whose occurrence represents the finishing of a treatment.

(2) Incorporation of the resources to each medical protocol. Resources are human beings (e.g., doctors, nurses), materials (e.g., x-ray machines, electrocardiogram machine) or other 


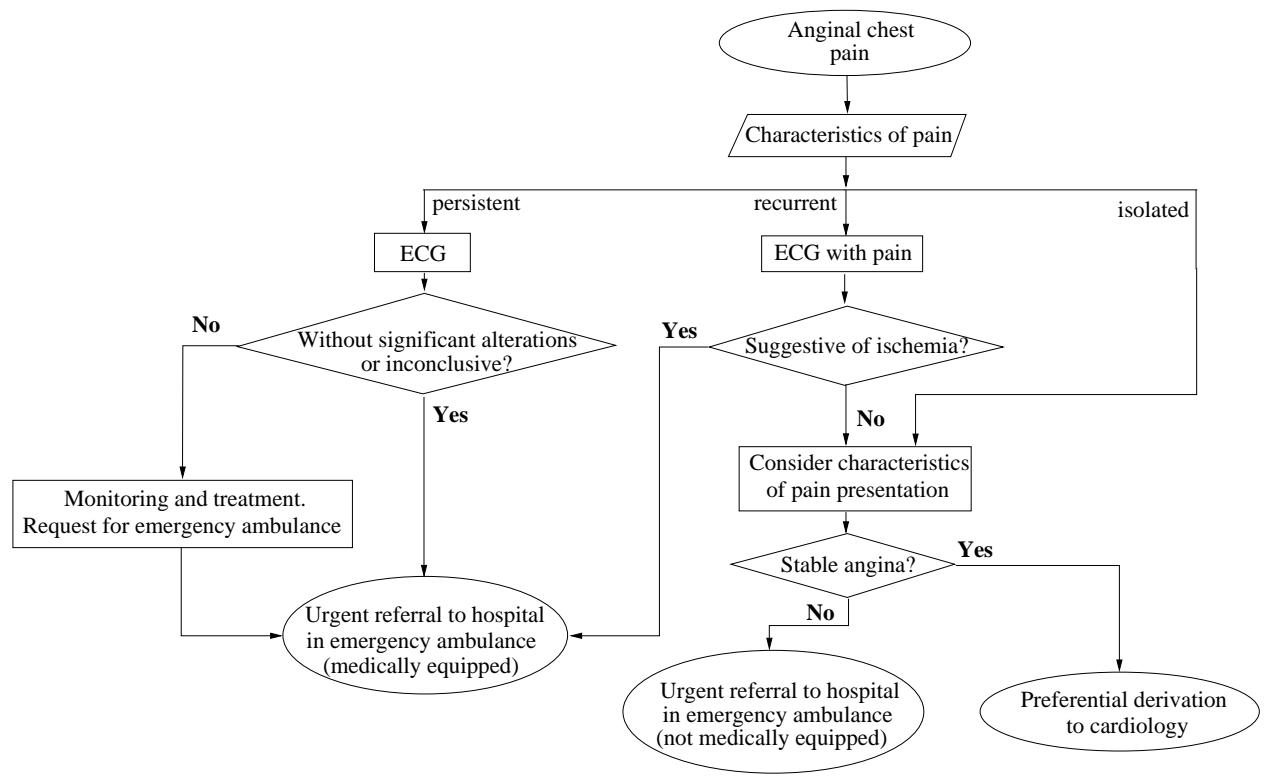

Fig. 1. A medical protocol for a patient with anginal chest pain in primary care team adapted from [5].

assets (e.g., beds, surgery rooms) that are needed to provide a diagnose and/or a treatment of a given protocol. All resources are of limited capacity and they are modeled by means of a place whose initial marking represents either the number of available copies of the resource (all copies are identical and any of the copies of a type of resource can satisfy a request) or its capacity (to meet simultaneously to several patients). A resource place has input (output) arcs to (from) those transitions of a medical protocol that moves a process to (from) a state that requires a number of copies of that resource type. In all cases, the considered resources can neither be created nor destroyed. However, the avability of the resources should not influence the decisions to be taken in the medical protocol.

(3) Construction of the global model by composition of the medical protocols with resources. In a health system there is a set of medical protocols, one per each disease and patient types. Nevertheless, resources are shared by different patients that follow different medical protocols. In order to obtain the global model of the health system we should compose the medical protocols with the needed resources. This composition is based on the fusion of the resource places representing the same resource type in different medical protocols. The initial marking of the resources, after the fusion, normally is computed as the maximum of the initial markings of the instances that have been merged.

A very similar methodology has been proposed for the modeling of manufacturing systems by Petri nets [6]. However, up to our knowledge, this is the first time that the proposed methodology is applied to a health system, obtaining Petri Nets with resources belonging to a known subclass of PNs. Notice that not all medical protocols can be modeled by this methodology. The models are restricted to systems where the resources are conservative. Moreover, in our present model a patient cannot die or be born. However, we believe that our methodology permits the modeling of a large number of health systems and many properties could be studied by exploiting the obtained model.

\section{HealthCare System Model}

This section defines a class of PNs to model healthcare systems following the methodology described in the introduction. Before introducing the formal definition of the class of nets let us consider a simple example.

Let us consider a simple medical protocol for a patient with anginal chest pain that is going to the primary care team (PCT) to be seen by his primary doctor. The protocol is adapted from [5] and it is shown in Fig. 1. The primary doctor should first do an anamnesis (rapid case history) to the patient and see the characteristics of his pain. If the pain is persistent (could be of hours or days with fluctuation of intensity), the primary doctor asks for an ECG in the PCT with the help of a nurse. Based on the results, the primary doctor could ask urgently for a medically equipped emergency ambulance and should start controlling and cure him until the emergency ambulance arrive. If the pain characteristic is recurrent (could be minutes but repeating in time) and the ECG with pain is suggestive of ischemic lesion the primary doctor asks for the emergency ambulance as well. If the ECG with pain is not suggestive of ischemia or the characteristic of the pain is isolated then the primary doctor should consider the pain presentation. If the angina is stable then the patient is sent to the specialized attention center to be seen by a specialist. Otherwise, he is sent to the hospital in an emergency ambulance but not necessary medically equipped.

The medical protocol in Fig. 1 that follows a primary care team (PCT) can be modeled by the Petri net in Fig. 2. Place 
$p_{P}$ is modeling the population assigned to the healthcare area where the PCT deploys the described protocol, i.e., the initial marking of this place is the maximal bound of active processes following simultaneously the protocol modeled by the net. If one patient has pain chest and is attending the PCT the output transition of $p_{P}$, i.e., $t_{0}$, is firing and one token in added to $p_{0}$. The number of tokens in $p_{0}$ represents the number of patients with anginal chest pain waiting to be seen by a primary doctor of the PCT. The firing rate of this transition $t_{0}$ can be conditioned by the statistical information (historic) of the incidence of the disease among the population of the area of deployment of the protocol. The initial diagnostic by the primary doctor is modeled by place $p_{1}$ and its markings is saying the number of patients that are seen in parallel by the primary doctors of the considered PCT. Depending on the initial diagnostic, the doctor decides to follow the protocol for the persistent pain (transition $t_{2}$ ), recurrent pain (transition $t_{3}$ ) or isolated pain (transition $t_{4}$ ). If the pain is persistent or recurrent, the first step is to make an ECG (place $p_{4}$ ) or an ECG with pain (place $p_{5}$ ). Notice that places $p_{2}$ and $p_{3}$ model the patients waiting for taking the ECG test. When this test is finished, the results are interpreted by the primary doctor $\left(p_{6}\right.$ or $p_{7}$ ).

The protocol of persistent pain follows by a choice in place $p_{6}$. If the ECG is without significant alterations or inconclusive (transition $t_{10}$ ) the patient is waiting for the ambulance (place $p_{9}$ ) to be transfer urgently to the hospital in an medically equipped emergency ambulance. On the contrary, (transition $t_{9}$ ) the primary doctor starts to cure him (place $p_{8}$ ) and asks for an emergency ambulance. In both situations, the patients are transferred to the hospital in the ambulance (place $p_{11}$ ) where they are cured and stabilized (place $p_{15}$ ) following the corresponding clinical guideline of the hospital.

In the case of recurrent pain and based on the results of the ECG with pain interpreted by the primary doctor there is a choice (in place $p_{7}$ ). If the ECG with pain is suggestive of ischemia the primary doctor requests an urgent transfer to the hospital in a medically equipped emergency ambulance (transition $t_{11}$ ). If the ECG with pain is not suggestive of ischemia (transition $t_{12}$ ) or the characteristic of pain is isolated (transition $t_{4}$ ) the primary doctor should consider the characteristics of pain presentation (place $p_{10}$ ) and will chose two possible future evolution. If the angina is stable (transition $t_{16}$ ) the patient is sent to be seen by a specialist (place $p_{13}$ ). If the angina is unstable (transition $t_{15}$ ), the patient is sent to the hospital (place $p_{12}$ is modeling the waiting for the ambulance) in an emergency ambulance but not necessary medically equipped (place $p_{14}$ ).

All places belonging to a medical protocol excepting the one modeling the population $\left(p_{P}\right.$ in our case) are (1) treatment places modeling treatments or cures that should be performed or (2) waiting places where the patients are waiting for the resource availability, e.g., $p_{0}$ modeling the patients waiting for a primary doctor. In order to receive a treatment or cure, one or more resources could be necessary. These resources are modeled in the PN model by places (depicted in blue

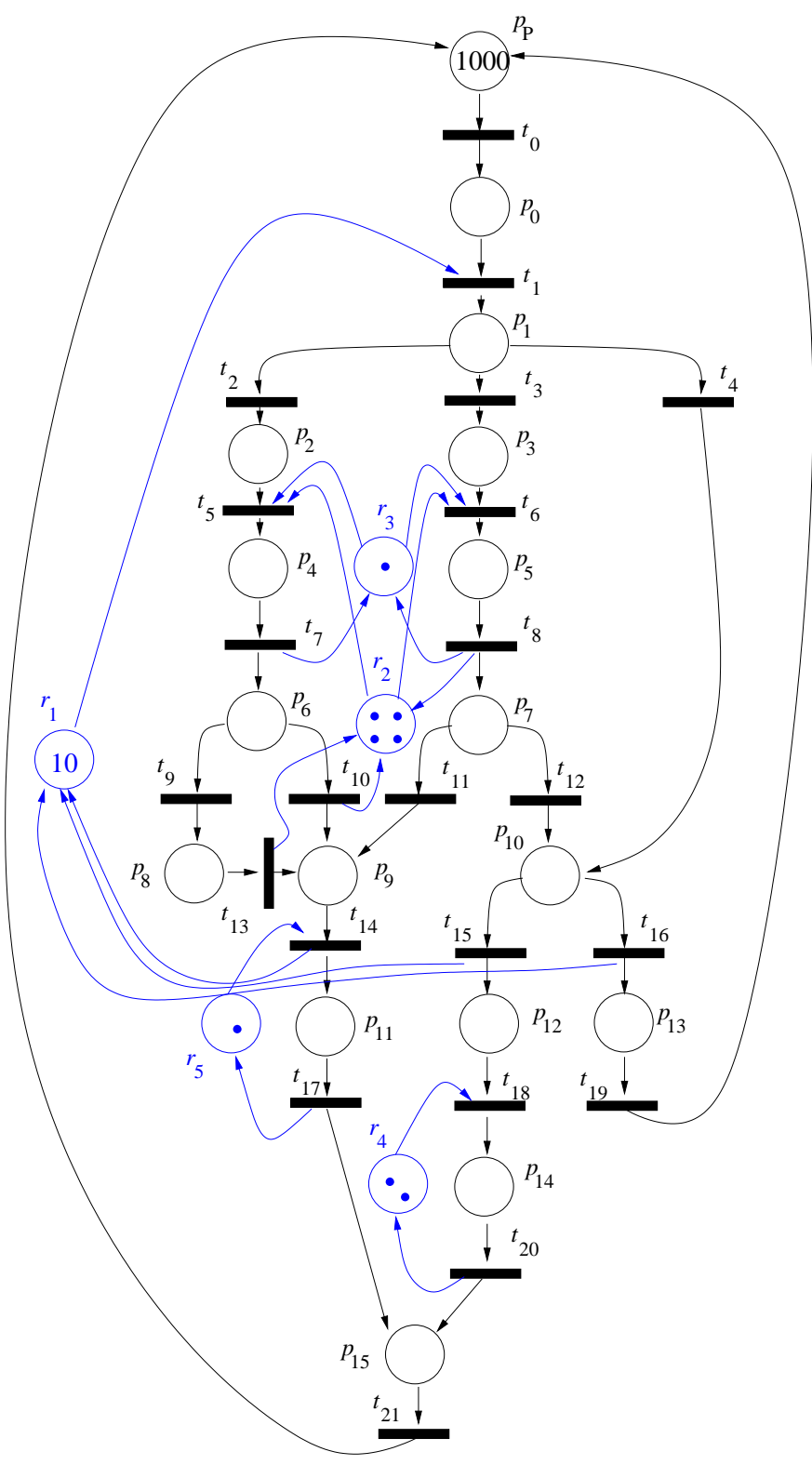

Fig. 2. Petri net model of the medical protocol of Fig. 1.

in Fig. 2) with an initial marking equal to the number of available instances of that resource. In our PN model, we have the following resources:

- $r_{1}$ is the place modeling the primary doctor resource. In this case we assume 10 primary doctors available for this medical protocol;

- $r_{2}$ models the nurses (a number of 4 nurses are assumed to be available);

- $r_{3}$ is the ECG machine that is unique in the PCT;

- the marking of $r_{4}$ represents the number of available emergency ambulances without medical equipment;

- $r_{5}$ is modeling the medically equipped emergency ambulances.

Now, let us give the formal definition of the PN class used to 
model a primary healthcare system.

Definition 1. Let $I_{\mathcal{N}}=\left\{1,2, \ldots,\left|I_{\mathcal{N}}\right|\right\}$ be a finite set of indices, where $\left|I_{\mathcal{N}}\right|$ is the number of medical protocols. $A$ healthcare Petri net $(H P N)$ is a connected $P N \mathcal{N}=$ $\langle P, T$, Pre, Post $\rangle$ where:

- $P=\left\{p_{P}\right\} \cup P_{0} \cup P_{S} \cup P_{R}$ is a partition such that:

- $p_{P}$ is the place models the healthy citizens in the area of the health system where protocols are deployed;

- $P_{0}=\bigcup_{i \in I_{\mathcal{N}}}\left\{p_{0_{i}}\right\}$ is the set of protocol waiting places. Each place $p_{0_{i}}$ models the patients in the healthcare system that should follow the protocol $i$;

- $P_{S}=\bigcup_{i \in I_{\mathcal{N}}} P_{S_{i}}, P_{S_{i}} \neq \emptyset$ and $P_{S_{i}} \cap P_{S_{j}}=\emptyset$ for all $i \neq j$ is the set of treatment and waiting places;

- $P_{R}=\left\{r_{1}, r_{2}, \ldots, r_{n}\right\}, n>0$ is the set of resource places.

- $T=\bigcup_{i \in I_{\mathcal{N}}} T_{i}, T_{i} \neq \emptyset$ and $T_{i} \cap T_{j}=\emptyset$ for all $i \neq j$;

- For all $i \in I_{\mathcal{N}}$, the subnet $\mathcal{N}_{i}$ generated by $P_{S_{i}} \cup\left\{p_{0_{i}}\right\} \cup$ $\left\{p_{P}\right\} \cup T_{i}$ is a strongly connected state machine ${ }^{1}$, such that every cycle $e^{2}$ contains $p_{P}$ and $p_{0_{i}}$. The subnet $\mathcal{N}_{i}$ is the PN model of the medical protocol $i$;

- For each $r \in P_{R}$ there exists a unique minimal $P$ semiflow, $\boldsymbol{y}_{r} \in \mathbb{N}^{|P|}$, such that $\{r\}=\left\|\boldsymbol{y}_{r}\right\| \cap P^{R}$, $\boldsymbol{y}_{r}[r]=1, p_{0_{i}} \notin\left\|\boldsymbol{y}_{r}\right\|$ for all $i \in I_{\mathcal{N}}, p_{P} \notin\left\|\boldsymbol{y}_{r}\right\|$ and $P_{S} \cap\left\|\boldsymbol{y}_{r}\right\| \neq \emptyset$;

- Pre $\in \mathbb{N}^{|P| \times|T|}$ is the pre-incidence matrix defining the weight of the arcs from places to transitions, i.e., Pre $[p, t]$ is the weight of the arc from $p$ to $t$;

- Post $\in \mathbb{N}^{|P| \times|T|}$ is the post-incidence matrix defining the weight of the arcs from transitions to places, i.e., Post $[p, t]$ is the weight of the arc from $t$ to $p$;

In a PN, places can contain a natural number of tokens. The amount of tokens in a place $p$ is denoted by $\boldsymbol{m}[p]$ and is called the marking of place $p$. The previous definition should be completed with the definition of the acceptable initial markings.

Definition 2. Let $\mathcal{N}=\left\langle\left\{p_{P}\right\} \cup P_{0} \cup P_{S} \cup P_{R}, T\right.$, Pre, Post $\rangle$ be a HPN. An initial marking $\boldsymbol{m}_{0}$ is acceptable for $\mathcal{N}$ if:

- $\boldsymbol{m}_{0}\left[p_{P}\right]>0$;

- for all $p \in P_{0} \cup P_{S}, \boldsymbol{m}_{0}[p]=0$;

- for all $r \in P_{R}, \boldsymbol{m}_{0}[r] \geq \max \left\{\boldsymbol{y}_{r}[p] \mid p \in\left\|\boldsymbol{y}_{r}\right\| \backslash\{r\}\right\}$ where $\boldsymbol{y}_{r}$ is the unique $P$-semiflow containing place $r$.

Notice that the HPN in Fig. 2 considers only the medical protocol for patients with anginal chest pain given in Fig. 1. Let us consider that the medical protocol for patients with low blood pressure should be implemented as well, hence added to the PN system. This medical protocol is sketched in Fig. 5 while the PN model of this medical protocol is given in Fig. 4. The global PN model is obtained by simple join/fusing place $p_{P}$ and resource places which are common in both PN protocols.

\footnotetext{
${ }^{1}$ A Petri net $\mathcal{N}$ is called state machine if all transitions has at most one input and one output place. If there is a path in $\mathcal{N}$ from any node (place or transition) to any other node than the net is strongly connected.

${ }^{2} \mathrm{~A}$ cycle is a closed path.
}

A patient with low blood pressure that arrives to the PCT (place $p_{0}^{\prime}$ ) is first attended by a nurse that measures his blood pressure $\left(p_{1}^{\prime}\right)$ and then is waiting to be seen by the doctor $\left(p_{2}^{\prime}\right)$. According to the value of the blood pressure and after the examination of the doctor $\left(p_{3}^{\prime}\right)$, the primary doctor could decide to ambulatory monitor the blood pressure $\left(t_{4}^{\prime}\right)$ or send the patient for an ECG $\left(t_{5}^{\prime}\right)$. If the ambulatory monitoring of the blood pressure is negative (the patient has a normal blood pressure during the 24 hours of monitoring with special equipment $\left.\left(r_{7}\right)\right)$ the patient is sent home $\left(p_{5}^{\prime} \rightarrow t_{7}^{\prime}\right)$. Otherwise, in $p_{7}^{\prime}$ waits for a doctor to be more investigated and in $p_{6}^{\prime}$ is waiting for an ECG. After this test (in $p_{8}^{\prime}$ ), if the primary doctor detects arrhythmia or heart failure (with signs of gravity) the patient is transfered to the hospital $\left(t_{12}^{\prime} \rightarrow p_{10}^{\prime}\right)$ in an emergency ambulance medically equipped $\left(p_{13}^{\prime}\right)$ where after his treatment $\left(p_{15}^{\prime}\right)$ he is sent home. If from the ECG the primary doctor cannot detect alterations the patient is sent for additional medical tests such as blood exam $\left(p_{9}^{\prime}\right.$ and $\left.p_{11}^{\prime}\right)$. Notice that the nurse should take a blood extraction from the patient and for this reason $r_{2}$ is assigned while resource $r_{6}$ is modeling the medical laboratory. The results of this analysis are evaluated by the primary doctor $\left(p_{14}^{\prime}\right)$. If the potassium is high and the sodium low $\left(t_{19}^{\prime}\right)$ with the probability of adrenal gland disorder the patient is referred to the endocrinology in the specialized attending center to be evaluated by a specialist $\left(p_{17}^{\prime}\right)$. If the blood test shows normal results $\left(t_{18}^{\prime}\right)$ the primary doctor make to the patient the orthostatic test - that is a measure of the blood pressure in decubitus and then orthostatic - $\left(p_{16}^{\prime}\right)$ and if it is negative send the patient home with medication $\left(t_{21}^{\prime}\right)$. Otherwise $\left(t_{22}^{\prime}\right)$ (positive test - means that the blood pressure decreases in orthostatism between certain values) the doctor is evaluating the possibility of cardiac syncope or carotid syncope $\left(p_{18}^{\prime}\right)$. If the test is positive $\left(t_{25}^{\prime}\right)$ then the patient is referred to the cardiology $\left(p_{20}^{\prime}\right)$. Otherwise, $\left(t_{24}^{\prime}\right)$ neurogenic causes should be considered by the doctor $\left(p_{19}^{\prime}\right)$. In the negative case $\left(t_{27}^{\prime}\right)$, the patient is sent home with medication (treatment in primary care team) while in the positive case - neurological causes $\left(t_{26}^{\prime}\right)$ the doctor should evaluate if the hypotension is incapacitating. If this result is negative (hypotension well tolerated) then the patient is sent home with medication $\left(t_{27}^{\prime}\right)$ while in the positive case is sent to the the neurologist $\left(p_{21}^{\prime}\right)$.

During the modeling of a medical protocol may be necessary to execute different operations in order to continue with the protocol. For example, the doctor can ask for different medical tests to be executed in different physical locations. It may seems that these are concurrent operations. In PN methodology, concurrent operations are modeled with a fork ${ }^{3}$ modeling the starting of the $\left|t^{\bullet}\right|$ treatments in parallel followed by a join ${ }^{4}$. If these constructions are used, the model will not be anymore a HPN since the subnet will not be anymore a state machine. However, in the case of the medical protocol of a patient with a particular disease the interleaving semantics

\footnotetext{
${ }^{3} t$ is a fork transition if $\left|t^{\bullet}\right|>1$, i.e., $t$ has more than one output place.

${ }^{4} t$ is a join transition if $|\bullet t|>1$, i.e., $t$ has more than one input place.
} 


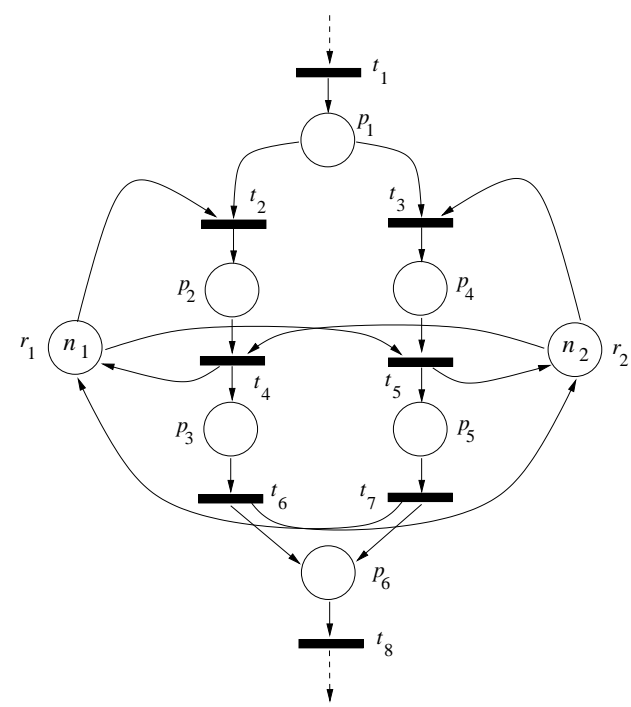

Fig. 3. Interleaving semantics of a medical protocol.

should be considered. This happens because the patient can do only one operation at a time moment and the decision on which one is executed first depends only on the existence of the corresponding resource.

Let us consider a small example where the doctor, in order to put a diagnostic, is sending the patient for a blood analysis and also for an ECG. Obviously, if the order in which these analysis should be performed is specified, the sequential operations are easy to model. Let us assume that the patient should perform first the analysis for which the required resource is available. The PN model is shown in Fig. 3. Assume that for the blood analysis a nurse is necessary for extraction. This resource is modeled by place $r_{1}$ where $n_{1}$ is the number of nurses available. Assume also that for the ECG is necessary the ECG machine modeled by place $r_{2}$. Notice that the transitions $t_{2}$ and $t_{3}$ are enabled, i.e., can be fired, depending on the marking of $r_{1}$ and $r_{2}$. If $\boldsymbol{m}\left[r_{1}\right]=0$, transition $t_{2}$ cannot be fired while if $\boldsymbol{m}\left[r_{2}\right]=0$, transition $t_{3}$ cannot be fired. The sequences $p_{2} \rightarrow p_{3}$ and $p_{4} \rightarrow p_{5}$ model the two possible orders in which the patient can take the analysis.

\section{PROPERTIES OF THE PN MODEL}

In this section, based on the Def. 1 and Def. 2 we give some basic properties of the HPN models.

Property 3 (Boundedness). $A H P N \mathcal{N}=\left\langle\left\{p_{P}\right\} \cup P_{0} \cup P_{S} \cup\right.$ $P_{R}, T$, Pre, Post $\rangle$ with an acceptable initial marking $\boldsymbol{m}_{0}$ is a bounded Petri net ${ }^{5}$.

Proof: According to Def. 1, each $\mathcal{N}_{i}, i \in I_{\mathcal{N}}$ generated by $P_{S_{i}} \cup\left\{p_{0_{i}}\right\} \cup\left\{p_{P}\right\}$ is a strongly connected state machine such that every cycle contains $p_{P}$ and $p_{0_{i}}$. Moreover, if $\boldsymbol{m}_{0}$ is an acceptable initial marking, the marking of all places of $\mathcal{N}_{i}$

\footnotetext{
${ }^{5} \mathrm{~A} \mathrm{PN}$ is bounded if the marking of each places is upper bounded at each reachable marking
}

is upper bounded by $\boldsymbol{m}_{0}\left[p_{P}\right]$. On the other hand, considering the resource places, since they are conservative, the places support of the corresponding $P$-semiflow are also bounded.

From the previous property is possible to conclude that: (1) the marking of the resource places is bounded by its corresponding initial markings; (2) the marking of the places $\left\{p_{P}\right\} \cup P_{0}$ is bounded by the number of citizens belonging to the healthcare system, i.e., initial marking of place $p_{P}$; (3) the marking of a place $p \in P_{S}$ is bounded by the minimal initial marking of the resource places modeling resources that an active process uses when it is in the place $p$ (if an active process in the place $p$ does not use resources, the marking of $p$ is bounded by the initial marking of place $p_{P}$ ).

Property 4 (PN class). A $H P N \mathcal{N}=\left\langle P=\left\{p_{P}\right\} \cup P_{0} \cup P_{S} \cup\right.$ $P_{R}, T$, Pre, Post $\rangle$ with an acceptable initial marking $\boldsymbol{m}_{0}$ and completed with a set of implicit places acting as resources of the Def. 1, belongs to the class of $S^{4} P R$ net systems.

Proof: This property trivially holds by adding a virtual resource place $p_{w}$ with an initial marking equal with $\boldsymbol{m}_{0}\left[p_{P}\right]$. Let $\bar{P}=\left\{P_{0} \cup P_{S}\right\} \backslash\left\{\bigcup_{r \in P_{R}}\left\{\left\|\boldsymbol{y}_{r}\right\|\right\}\right\}$, where $\boldsymbol{y}_{r}$ is the unique $\mathrm{P}$-semiflow corresponding to resource $r$ according to Def. 1. Places from $\bar{P}$ belong to a medical protocol but does not require the previous allocation of any resource, i.e, are waiting places. However, we can always allocate to these places a virtual resource $p_{w}$ by connecting accordingly $p_{w}$ with their input and output transitions. Since $\boldsymbol{m}_{0}\left[p_{w}\right]=\boldsymbol{m}_{0}\left[p_{P}\right]$, place $p_{w}$ will be an implicit place, i.e., will not change the behavior of the system if it is removed. In the resulted net, all treatment and waiting places will require at least one resource. Therefore, taking as the set of idle places the place $p_{P}$, it can be easily check that the resulted net is a $S^{4} P R$ net system [6].

According to Prop. 4, all results of $S^{4} P R$ nets also hold for HPN systems. This is because the needed places to transform the HPN in a $S^{4} P R$ always are implicit places. The following properties are adapted from $S^{4} P R$ net systems [6] to the HPN framework.

Let us consider the PN in Fig. 2 and the decision that should be made at $p_{1}$ after the first anamnesis of the primary doctor. The next state should depend only on this anamnesis (in this case on the characteristics of the pain) and not on the availability of the ECG machine. Therefore, for the first two choices for which is necessary the ECG machine two intermediate places are introduced $\left(p_{2}\right.$ and $\left.p_{3}\right)$ to model the waiting queues avoiding the assignment of resource $r_{3}$ at $t_{2}$ and $t_{3}$.

Property 5 (Cycles of a medical protocol). Let $\left\langle\mathcal{N}, \boldsymbol{m}_{0}\right\rangle$ be a HPN system. All cycles of $\mathcal{N}$ containing $p_{P}$ are minimal $T$ semiflow ${ }^{6}$. If $\boldsymbol{m}_{0}$ is acceptable then any cycle can be executed

\footnotetext{
${ }^{6} \mathrm{~A}$ vector $\boldsymbol{x} \geqslant 0$ is a T-semiflow of a PN $\mathcal{N}=\langle P, T$, Pre, Post $\rangle$ if $(\boldsymbol{P o s t}-\boldsymbol{P r e})^{+} \cdot \boldsymbol{x}=0$. A T-semiflow is called minimal if the greatest common divisor of its elements is equal to one and no subset of its support $\|\boldsymbol{x}\|$ is also a T-semiflow, where the support of a vector is the set of indices of positive elements.
} 
in isolation.

Proof: A cycle of $\mathcal{N}$ containing $p_{P}$ is cycle in the state machine $i$, i.e., the subnet considering only the set of places $\left\{p_{P}\right\} \cup\left\{p_{0_{i}}\right\} \cup P_{S_{i}}$. In a state machine $\mathrm{PN}$, any cycle corresponds to a T-semiflow $\boldsymbol{x}$ [7]. According to Def. 1 resources are conservative, therefore $\boldsymbol{x}$ is also a T-semiflow of the whole net. If $\boldsymbol{m}_{0}$ is acceptable then the marking of $p_{P}$ is strictly positive and any T-semiflow can be fired.

For the PN model in Fig. 2, one cycle is given by: $p_{P}$, $t_{0}, p_{0}, t_{1}, p_{1}, t_{2}, p_{2}, t_{5}, p_{4}, t_{7}, p_{6}, t_{9}, p_{8}, t_{13}, p_{9}, t_{14}, p_{11}$, $t_{17}, p_{15}, t_{21}$. Notice that the transitions of this path can be fired indefinitely in this order and one repetition leaves the initial marking unchanged. In fact, this represents a possible sequence of treatments and cures that should be followed by a patient with anginal chest pain.

Property 6 (Consistency of the HPN). Let $\left\langle\mathcal{N}, \boldsymbol{m}_{0}\right\rangle$ be a HPN system modeling a medical protocol. $\mathcal{N}$ is covered by T-semiflows or equivalently, it is consistent.

Proof: This property holds according to Prop. 5 and based on the following observation: for all $i \in I_{\mathcal{N}}$ the subnets generated by $P_{S_{i}} \cup\left\{p_{0_{i}}\right\} \cup\left\{p_{P}\right\} \cup T$ are strongly connected state machines and all resources are conservative.

In the PN terms, a T-semiflow corresponds to a potential repetitive behavior. According to Prop. 5, any cycle corresponds to a T-semiflow of the net. Consequently, the Tsemiflows computed in the PN model can be very useful for the verification of the model and also of the medical protocol. For example, a T-semiflow of the protocol path (cycle) given before is a vector of dimension 21 having all elements equal to zero excepting the ones corresponding to: $t_{0}, t_{1}, t_{2}, t_{5}, t_{7}$, $t_{9}, t_{13}, t_{14}, t_{17}, t_{21}$ that are equal to one.

Because a marked strongly connected state machine can move all tokens to any place of the net, the following observation holds: given an intermediate execution state of a medical protocol, it is always possible to terminate recovering the initial state.

Property 7 (Conservativeness of the HPN). Let $\left\langle\mathcal{N}, \boldsymbol{m}_{0}\right\rangle$ be a HPN system modeling a medical protocol and $\boldsymbol{m}_{0}$ an acceptable marking. $\mathcal{N}$ is conservative.

Proof: Let us consider the net obtained by adding the virtual resource place $p_{w}$ as in Prop. 4. In the resulted net, all partial states of a medical protocol use some resources to carry out the treatments they represent. Therefore, the resulted net is conservative implying that the original HPN net systems is conservative.

For example, place $p_{4}$ in PN of Fig. 2 models a patient making an ECG. For this operation, the following resources are necessary: ECG machine $\left(r_{3}\right.$ assigned at $\left.t_{5}\right)$, a nurse $\left(r_{2}\right.$ assigned at $\left.t_{5}\right)$ and a primary doctor $\left(r_{1}\right.$ assigned at $\left.t_{1}\right)$.

\section{EXPLOITING THE PN MODEL}

In this section we show that by exploiting the PN model of the two medical protocols described in the paper can be used to obtain useful information about the real system.

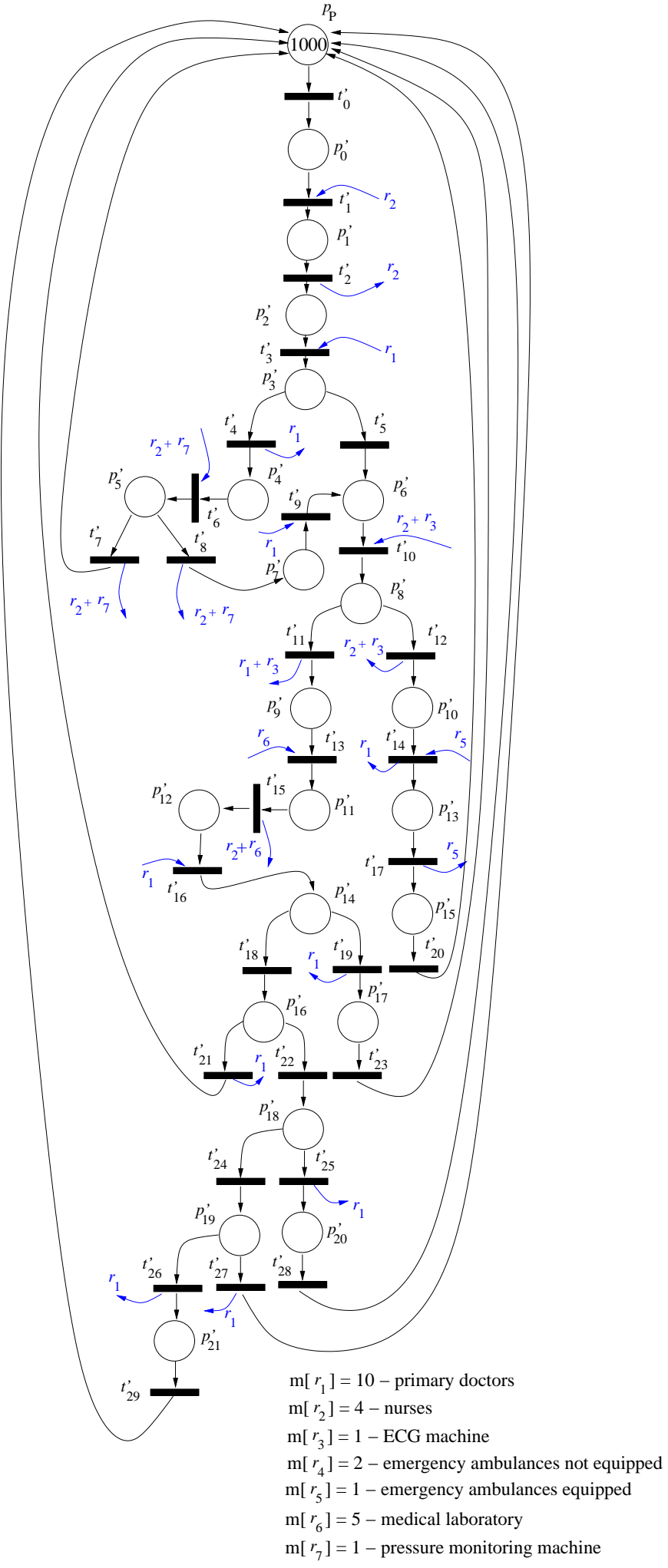

Fig. 4. Petri net model of the medical protocol of Fig. 5. The resource places are not represented for sake of clarity. The allocations and releases of the resources are shown by blue arcs. The initial marking of the resources is given in the right-bottom corner. 


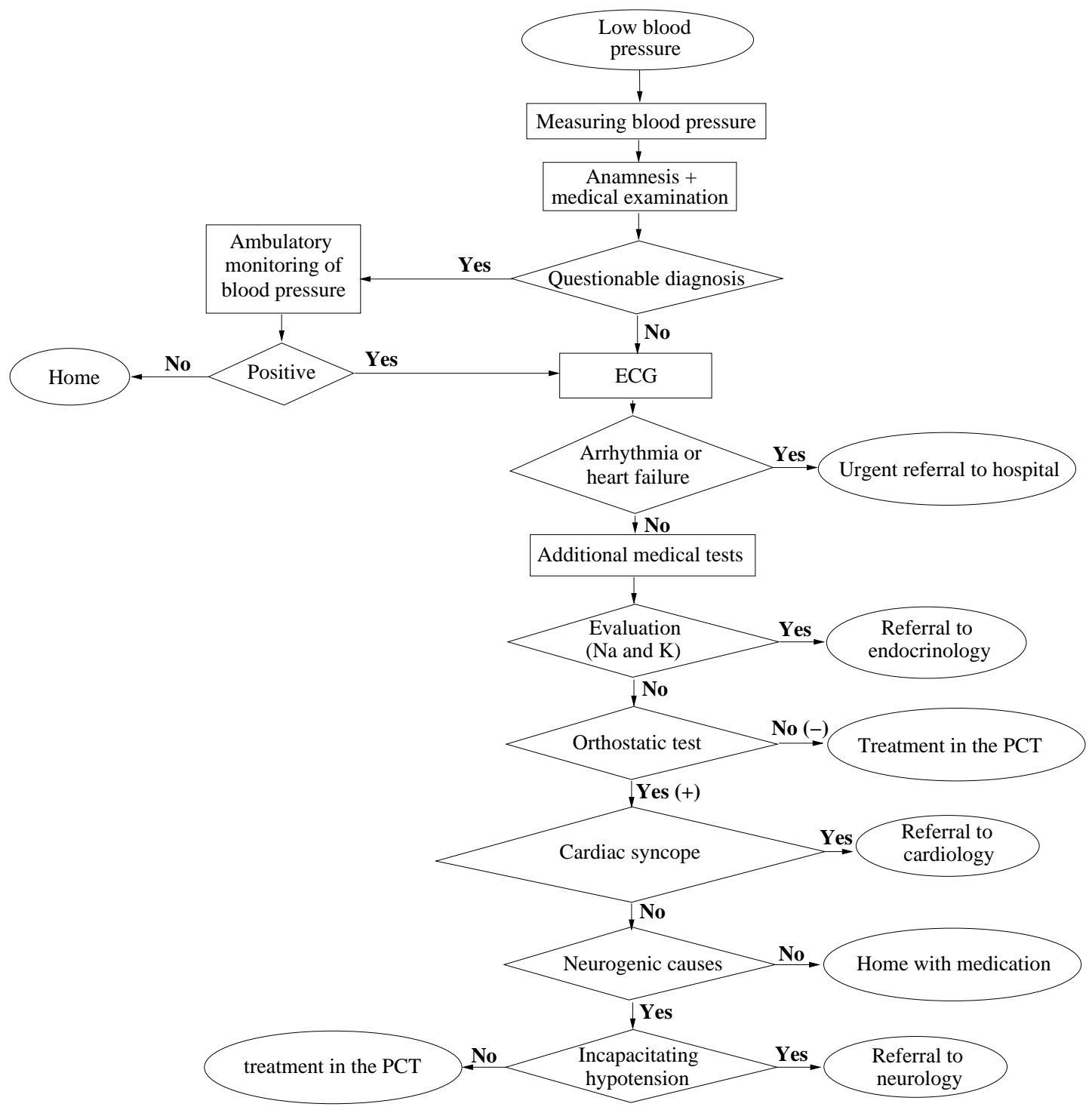

Fig. 5. A medical protocol for a patient with low blood pressure in primary care team adapted from [5].

P-semiflows. This net system has 8 minimal P-semiflows, 7 of them corresponding to the conservation of the resources and 1 corresponding to the conservation of the PCT citizens. For example, the P-semiflow corresponding to the primary doctors is:

$$
\begin{aligned}
& p_{1}+p_{2}+p_{3}+p_{4}+p_{5}+p_{6}+p_{7}+p_{8}+p_{9}+p_{10}+ \\
& +p_{3}^{\prime}+p_{6}^{\prime}+p_{8}^{\prime}+p_{10}^{\prime}+p_{14}^{\prime}+p_{16}^{\prime}+p_{18}^{\prime}+p_{19}^{\prime}+r_{1}=10
\end{aligned}
$$

This means that the number of primary doctors is constant at each time moment equal to 10 . Moreover, using the P-semiflow we can identify the treatments and cures of the protocols that is requiring a primary doctor. In this case, the places belonging to the low blood pressure protocol are: $p_{3}^{\prime}$ (anamnesis and medical examination by the primary doctor); $p_{6}^{\prime}$ (the patient is under the supervision of the doctor waiting for the ECG); $p_{8}^{\prime}$ (the doctor evaluates the results of the ECG); $p_{10}^{\prime}$ (the primary doctor detects arrhythmia or heart failure and is monitoring the patient until the ambulance arrives); $p_{14}^{\prime}$ (evaluation of the medical analysis); $p_{16}^{\prime}$ (performing an orthostatic test); $p_{18}^{\prime}$ (evaluation of a cardiac or carotid syncopes) and $p_{19}^{\prime}$ (consideration of neurogenic causes).

Time model. In the healthcare systems are of interest the properties that depend on time, i.e., quantitative properties. For this reason, temporal specification should be introduced to the PN model. In the PN literature, the time can be associated to transitions or to places. For our application, since a treatment or a cure is modeled by a place we will assign time to places. Each token that will arrive to a place will be delayed in the place for a time duration (for example is the time necessary to finish a treatment or a cure). These delays can be random variables, for example with negative probability distribution function, or constant delays.

Let us consider that all treatment places $P_{T}$ have assigned 
exponential time delays with rates $\lambda_{i}=1$ (i.e., all treatments and cures are executed in average in 1 time unit) for the PN models in Fig. 2 and Fig. 4. To place $p_{P}$ we associate an exponential time delay with rate $1 / 100$ meaning that if a patient is cured, will became ill again, in average, after 100 time units. To places $p_{0}$ and $p_{0}^{\prime}$ we associate an exponential time delay with rate $1 / 5$ while the conflict between $t_{0}$ and $t_{0}^{\prime}$ is probabilistic with the probability of $t_{0}$ equal to 0.3 while the probability of $t_{0}^{\prime}$ is equal to 0.7 . Assume that for all other conflict transitions the same probability of firing is assigned except for the transitions where the doctor is allocated. To these transitions, i.e., $t_{1}, t_{3}^{\prime}, t_{9}^{\prime}$ and $t_{16}^{\prime}$ we assign priorities because we want to assign the doctor firstly to the patient that was already consulted. Hence, we assume that $t_{16}^{\prime}$ has biggest priority, followed by $t_{9}^{\prime}, t_{1}$ and finally $t_{3}^{\prime}$. This is not a very realistic scenario but the proposal of the following simulations is to show the advantages of having a formal model of the system. Many performance indices can be predicted and these models can be used when decisions should be taken.

The PN system is used to make one replica by simulation using Petri net Toolbox [8] for 1000 time units and the following results are obtained:

- Number of patients with anginal chest pain cured: 296 (no of firings of $\left.t_{21}\right)+108$ (number of firings of $t_{19}$ ) = 404 patients from 413 arrived at PCT;

- Number of patients with low blood pressure cured: 204 $\left(t_{7}^{\prime}\right)+311\left(t_{2^{\prime}}^{\prime}\right)+86\left(t_{21}^{\prime}\right)+179\left(t_{23}^{\prime}\right)+42\left(t_{28}^{\prime}\right)+27$ $\left(t_{29}^{\prime}\right)=849$ from 1051 arrived at PCT;

- Average number of patients with anginal chest pain waiting for an emergency ambulance (average number of tokens in $\left.p_{12}\right): 0.62$ while the time between two patients that arrive to the queue (average time between two arrival of tokens in $p_{12}$ ): 2.75 ;

The following experiment is obtained by incrementing the number of tokens in $r_{1}$ from 10 to 20 and see how many patients are cured. The results are shown in Fig. 6 and can be observed that practically the number of patients are the same in all cases. Therefore, incrementing the number of primary doctors is not a good decision if only these two medical protocols are considered.

\section{COnClusion}

In this paper we define a new class of Petri net used for modeling medical protocols and the resources they need to progress in primary healthcare systems. We have defined a methodology for the systematic construction of the model. The first step is to model the sequences of treatments and cures that the patient should follow for a particular disease. From these sequences the medical protocol for the disease is obtained in the form of a Petri Net belonging to the class of state machines. In the second step, the resources needed in each stage of a protocol are added to the model and finally, the PNs of different medical protocols are merged by fusing the common resource places and the full model is obtained. We have proven that the obtained nets are essentially $S^{4} P R$ net systems, except for some implicit places that could be needed

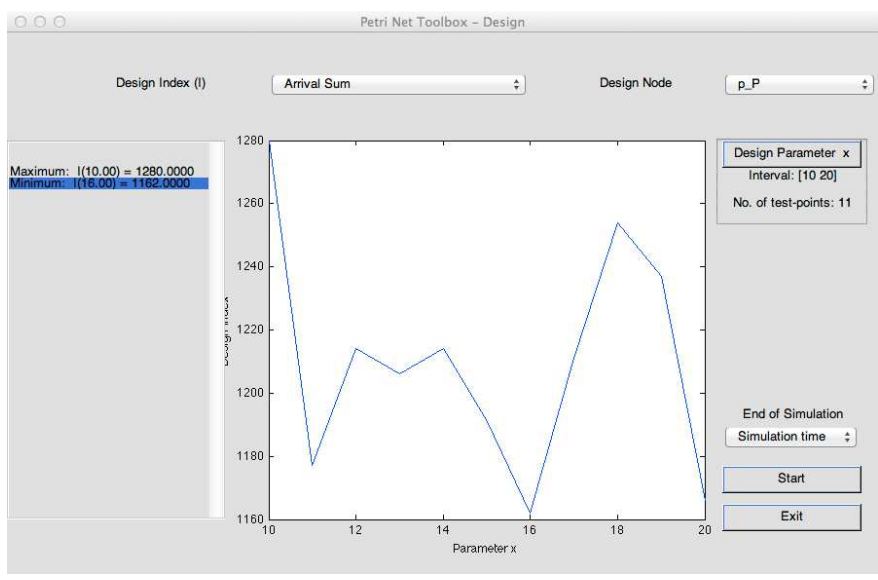

Fig. 6. Sketch of a PN Toolbox window illustrating a design experiment. Assuming the number of doctors of the PCT varying from 10 to 20 the number of cured patients is shown for each case.

to complete the HPN, and therefore the HPN class inherits the properties list and their characterization from the $S^{4} P R$ class. Some basic properties of the HPN class are given and at the end, some simulations results show how they can be used to obtain different performance indices of the healthcare system by simulations. To illustrate the full viability of the approach we have presented a realistic example integrating two medical protocols.

\section{REFERENCES}

[1] M. Lamiri, X. Xie, A. Dolgui, and F. Grimaud, "A stochastic model for operating room planning with elective and emergency demand for surgery," European Journal of Operational Research, vol. 185, no. 3, pp. 1026-1037, 2008.

[2] M. Lamiri, X. Xie, and S. Zhanga, "Column generation approach to operating theater planning with elective and emergency patientsy," IIE Transactions, vol. 40, no. 9, pp. 838-852, 2008.

[3] M. Dotoli, M. Fanti, A. Mangini, and W. Ukovich, "A continuous Petri net model for the management and design of emergency cardiology departments," in ADHS09: Proc. of the 3rd Analysis and Design of Hybrid Systems, Zaragoza, Spain, 2009.

[4] C. Mahulea, J.-M. García-Soriano, and J.-M. Colom, "Modular Petri net modeling of the Spanish Health System,' in ETFA'2012: 17th IEEE International Conference on Emerging Technologies and Factory Automation, Krakow, Poland, 2012, pp. 1-8.

[5] J. Boquet, Ed., Guía de Ayuda al Diagnóstico en Atención Primaria. Sociedad Española de Medicina de Familia y Comunitaria, 2005.

[6] J. M. Colom, "The resource allocation problem in flexible manufacturing systems," in Applications and Theory of Petri Nets, ser. Lecture Notes in Computer Science, W. Aalst and E. Best, Eds. Berlin, Heidelberg: Springer-Verlag, 2003, vol. 2679, pp. 23-35.

[7] T. Murata, "Petri nets: Properties, analysis and applications," Proceedings of the IEEE, vol. 77, no. 4, pp. 541-580, 1989.

[8] M. Matcovschi, C. Mahulea, and O. Pastravanu, "Petri Net Toolbox for MATLAB," in MED03: 11th IEEE Mediterranean Conference on Control and Automations, Rhodes, Greece, 2003. 